\title{
Diagnosis and treatment of chronic rhinosinusitis: focus on intranasal Amphotericin B
}

\author{
Eugene B Kern' \\ David Sherris' \\ Angelos M Stergiou ${ }^{2}$ \\ Laura M Katz ${ }^{2}$ \\ Lisa C Rosenblatt ${ }^{3}$ \\ Jens Ponikau' \\ 'Department of Otorhinolaryngology, \\ University at Buffalo, The State \\ University of New York, NY; \\ ${ }^{2}$ Accentia Biopharmaceuticals, New \\ York, NY; ${ }^{3}$ Analytica International, \\ New York, NY, USA
}

\begin{abstract}
Chronic rhinosinusitis (CRS) is a chronic disease that affects $14.2 \%$ of the US adult population. Despite being widespread, little is known about the etiology of CRS. Treatment has been symptomatic and focused on relieving symptoms. Recent investigations into causes of CRS have revealed that most CRS patients have an eosinophilic infiltration of their nasal tissue (mucosa), regardless of atopy and elevated immunoglobulin E levels. Although fungi are ubiquitous and in the nasal mucus of both healthy people and patients, it is only in the patients that the eosinophils (part of the inflammatory response) are found. Fungi in the nasal mucus are harmless, yet in CRS patients these same fungi stimulate an inflammatory response, inducing the eosinophils to leave the blood vessels and enter the nasal and sinus tissue and ultimately enter the nasal airway mucus. In the nasal mucus these eosinophils attack the fungi and destroy the fungi by the release of a toxic substance called major basic protein (MBP) from the granules in the eosinophils. This degranulation and release of the toxic MBP not only destroys fungi, but also produces collateral damage injuring the nasal and sinus mucosal lining tissue. The injury to the mucosal lining makes the nasal and sinus mucosa susceptible to penetration and potential infection by bacteria. When this tissue inflammation and damage is persistent and prolonged we call it CRS. The diagnosis of CRS is based largely on symptomatic criteria, with anterior rhinoscopy or endoscopy, and, if there is any doubt about the diagnosis, computed tomography imaging is employed to confirm the presence of diseased sinus mucosa. Treatment of CRS, whether medical (intranasal corticosteroids, saline irrigations) or surgical, is aimed at decreasing inflammation and obstruction in the sinonasal passages. Antibiotics, although commonly used in CRS, should not be administered unless there is suspicion of an acute bacterial infection. The theory behind the fungal and eosinophilic etiology of CRS has led to use of an antifungal compound, intranasal Amphotericin B. In clinical studies, topical irrigation with Amphotericin B has been shown to be both a safe and effective treatment for CRS.
\end{abstract}

Keywords: chronic sinusitis, rhinosinusitis, diagnosis, treatment, antifungal, Amphotericin B

\section{Introduction}

Chronic rhinosinusitis (CRS) is a chronic disease that involves long-term inflammation of the nasal and paranasal sinus mucosa (Benninger et al 2003). It is an extremely common condition, affecting 29.2 million (14.2\%) US adults (Lethbridge-Çejku et al 2004, 2006). Although commonly known as 'chronic sinusitis', the term 'chronic rhinosinusitis' is now being used more frequently, due to the involvement of the entire nasal and sinus passages seen with this condition (Benninger et al 2003). CRS causes not only physical suffering, but also impacts psychological wellbeing and daily functioning. CRS is estimated to result in an annual 18 to 22 million physician office visits in the US (Benninger et al 2003).

Despite its widespread prevalence and substantial impact on the population, there are no Food and Drug Administration-approved drugs for the management and treatment of CRS. This is due largely to the lack of research into safe and effective
Correspondence: Angelos M Stergiou Accentia Biopharmaceuticals, 450 Park Avenue South, $12^{\text {th }}$ Floor, New York, NY 10016, USA

$\mathrm{Ph}+12126864100 \times 8101$

$\mathrm{Fax}+12126868601$

Email astergiou@accentia.net 
treatments, which in turn is related to the lack of understanding of the pathophysiology of the disease (Meltzer et al 2004). This review will focus on current thinking and evidence for the pathogenesis of CRS, as well as current diagnostic methods and treatment options, including the topical intranasal use of antifungal compounds.

\section{Clinical definition}

The term CRS encompasses all inflammatory disorders of the nose and paranasal sinuses with a minimum duration of 12 weeks. This definition was put forth in 1996 by the Rhinosinusitis Task Force (RSTF), which was established by the American Academy of Otolaryngology - Head and Neck Surgery (AAO-HNS) (Benninger et al 2003). However, this definition does not include a specific etiology for the eosinophilic inflammatory process.

\section{Etiology and pathogenesis}

CRS has a number of proposed causes. It is often attributed to bacterial infection; a variety of aerobic (staphylococcus spp., Gram-negative rods) and anaerobic (prevotella, fusobacterium, peptostreptococcus) bacteria have been cultured from patients with CRS (Meltzer et al 2004). However, it is unclear if bacteria are causing infection, are exposing the host to superantigens causing an inflammatory response, or are able to colonize due to pre-existing pathology of the sinus mucosa (Meltzer et al 2004).

A fairly recent concept in the pathogenesis of CRS is colonization with fungi. Due to the presence of fungal spores in the air, fungus is a common finding in the upper respiratory tract even of healthy individuals. There are, however, clinical subentities of CRS that have long been attributed to fungal etiology. Allergic fungal rhinosinusitis (AFRS) is a type of CRS in which patients have an allergic response to the fungus colonizing the mucin in their sinonasal cavities. In contrast, fungus balls are caused by overgrowth of fungus in the nose and paranasal sinuses, without an inflammatory reaction (Meltzer et al 2004). The inflammatory reaction in response to a fungus ball is more of an irritative inflammation, like a foreign body reaction, ie, giant cells, and not an eosinophilic inflammation, which is present in CRS.

Recent studies have attributed a much broader role to fungi in CRS. It is postulated that in certain individuals, colonizing fungi provoke a hypersensitivity response that is non-immunoglobulin $\mathrm{E}$ (IgE)-mediated. Rather than an allergic response, the fungi stimulate a local inflammatory response with infiltration of eosinophils. This condition has been termed eosinophilic fungal rhinosinusitis (EFRS), and it has been implicated in the majority of cases of CRS (Meltzer et al 2004).

The immune response in CRS patients is commonly a partial Th2 lymphocyte response (production of interleukin [IL]-5, IL-13, a small amount of IL-4); this is the immune profile seen even among CRS patients who have a positive skin response to fungal allergens, where one would expect increased IL-4, as it is necessary for IgE synthesis (Hamilos and Lund 2004) In fact, it seems that a universal immune response in CRS is a T-cell response (a mix of Th1 and Th2 cells) with IL-5 as the most recognized mediator, IL-13, and little IL-4 (Hamilos and Lund 2004). Although allergic rhinitis can be present as a comorbid condition, the patient with CRS alone exhibits eosinophilic mucin on histology, without evidence for IgE-mediated allergy (Sasama et al 2005).

The mucin of CRS patients also contains fungal hyphae. Ponikau and colleagues (1999) collected specimens from 210 patients with CRS (with or without polyposis) and found that $96 \%$ were culture positive for multiple fungi, the most common being Alternaria spp. (44.3\%). Interestingly, 100\% of the 14 healthy controls also had positive fungal cultures, with similar mycology. Other recent studies have found similar results using fungal DNA detection with PCR (Gosepath et al 2004). Therefore fungi are present in patients with CRS and in healthy controls. The difference between the CRS patients and the controls in this study was the presence of eosinophils in tissue specimens (96\% of 101 CRS surgical cases vs none of 4 controls). Fungi, only in sensitized individuals, will initiate the eosinophilic reaction, which targets the fungi in the mucous and allows the degranulation of the eosinophils.

The authors hypothesized that the patients with CRS were forming an eosinophilic reaction toward the fungal hyphae in their sinonasal mucin; the eosinophils migrate through the epithelium and degranulate in the mucin, causing the inflammatory response (Ponikau et al 1999). It is likely that it is the fungal antigen itself in patients with CRS, which is able to induce migration and degranulation of the eosinophils. This fungal antigen is derived from the germinating fungal spores. Thus, it is the airborne fungal spores, not a fungal colony itself which is required to induce the immunologic cascade. Eosinophilic degranulation is thought to cause the local tissue damage seen in CRS, mainly through release of major basic protein (MBP). It appears that MBP itself inflicts the epithelial damage directly because of its toxicity (Frigas et al 1980; Harlin et al; Motojima et al 
1989). The airway remodeling, with basement membrane thickening and epithelial damage, is similar to that seen from the eosinophilic inflammatory process in asthma (Ponikau et al 2003). In contrast to allergic rhinitis, in which the eosinophils do not degranulate, they release only scant amounts of MBP and they do not target fungi.

Meltzer and colleagues' December 2004 definitions subcategorized patients into those with and those without polyps (Meltzer et al 2004). Meltzer and colleagues suggested that patients without polyps may be a subcategory of CRS in which the inflammatory cells in the mucin are mostly neutrophils. Cases with polyps, however, were noted to have eosinophilic inflammation. In contrast, Ponikau and colleagues noted that, regardless of the presence or absence of polyps, mucin from patients with CRS uniformly have substantially elevated levels of MBP, a well-accepted surrogate marker for eosinophilic migration and degranulation (Ponikau, Sherris, Kephart, et al 2005). One might postulate that, over time in CRS patients, this chronic and debilitating disease with accumulation of mucosa may result in polyps.

The common histopathologic denominator in CRS with, and in some cases without, nasal polyposis, therefore, is the eosinophilic infiltration into the nasal mucosa. This is the histological hallmark of CRS in that the eosinophils are present independently from atopy and are nearly absent in healthy controls (Ponikau et al 1999). It is commonly accepted that the role of eosinophils in the immune system is the defense against large, nonphagocytosable pathogens such as helminthic parasites (Gleich et al 1993).

\section{Diagnosis}

It is important to recognize the signs and symptoms associated with the disease to be able to arrive at a diagnosis of CRS. Through a careful patient history, the clinician should be able to collect information on timing and severity of symptoms associated with CRS (Meltzer et al 2004). The RSTF developed a list of major and minor criteria (signs and symptoms) to aid in diagnosis of rhinosinusitis (Table 1). CRS is probable if the patient has two or more major factors or one major and two or more minor factors for more than 12 weeks; CRS should be considered in the differential diagnosis if the patient has one major factor or two or more minor factors for more than 12 weeks (Benninger et al 2003).

It is also important to gauge the severity of the condition in the patient as well as the impact on the patient's quality of life (QoL). A number of severity scoring systems and QoL questionnaires have been developed for assessment of
CRS (Meltzer et al 2004). Quantifying the impact of CRS on a patient's daily functioning aids in determining future patient outcomes with given treatments (Meltzer et al 2004).

In addition to history, physical signs are important criteria in the diagnosis of CRS. Anterior rhinoscopy is a noninvasive means of viewing the nasal mucosa (Meltzer et al 2004). However, it is difficult to view beyond the anterior portion of the nasal passages, even after administration of topical decongestants. Nasal endoscopy, although more invasive, is a preferable method for obtaining a magnified view of the nasal mucosa, turbinates, and interior of the nasal airway in the preoperative and postsurgical patient. Endoscopy aids in assessing the integrity of the mucosa, as well as directly seeing mucosal changes, polyps, crusting, and/or discharge. Cultures can also be obtained endoscopically (Meltzer et al 2004). Discolored nasal discharge, polyps, or polypoid swelling seen with anterior rhinoscopy or endoscopy, and edema or erythema of the middle meatus or ethmoid bulla seen on endoscopy, are consistent with CRS (Benninger et al 2003). If edema, erythema, or granulation tissue do not extend to the middle meatus or ethmoid bulla, radiologic imaging is necessary to view the sinuses and to make or confirm the diagnosis of CRS (Benninger et al 2003).

Imaging modalities are often necessary in CRS, especially in patients with refractory or recurrent disease. Plain film X-rays have not been proven to be useful in CRS. Computed tomography (CT) scanning is the imaging method of choice. Direct coronal CT affords an excellent view of the bony structures and mucosal lining. These coronal CT scans are indicated in patients with recurrent sinusitis or CRS in order to properly consider treatment options and sequence the steps in the evaluation. In surgical candidates,

Table I Factors associated with diagnosis of rhinosinusitis (RSTF I996) (Benninger et al 2003)

\begin{tabular}{ll}
\hline Major factors & Minor factors \\
\hline Facial pain/pressure* & Headache \\
Nasal obstruction/ blockage & Fever (all nonacute rhinosinusitis) \\
Nasal discharge/ purulence/ & Halitosis \\
discolored postnasal drainage & Fatigue \\
Hyposmia/ anosmia & Dental pain \\
Purulence in nasal cavity on & Cough \\
examination & \\
Fever (acute rhinosinusitis only) & Ear pain/ pressure/ fullness \\
\hline
\end{tabular}

Note: *Facial pain/pressure alone does not constitute a suggestive history for rhinosinusitis in the absence of another major nasal symptom or sign; ${ }^{\dagger}$ Fever in acute sinusitis alone does not constitute a strongly suggestive history for rhinosinusitis in the absence of another major nasal symptom or sign. 
CT scanning clearly defines the surgical anatomy and the extent of the disease process (Meltzer et al 2004) (Figures 1 and 2). Mucosal thickening, bony changes, or air-fluid levels seen on CT are consistent with CRS (Benninger et al 2003). There are instances when lateral or axial imaging is required to determine the extent of the disease process. Magnetic resonance imaging (MRI) is not recommended for diagnosis of CRS due to its lack of specificity (Benninger et al 2003), however, it is superior to CT for differentiation between infectious (bacterial or viral) inflammation and fungal concretions as well as in detecting malignancies (Meltzer et al 2004). MRI can also detect extension of disease beyond paranasal sinuses into the orbital region and intracranial compartment (Benninger et al 2003).

Laboratory tests, such as nasal cytology, nasal biopsy, and hematologic analyses are not necessary for the clinical diagnosis of CRS. They can aid in determining whether other conditions are present, such as acute bacterial infection or allergy, or more serious conditions such as cystic fibrosis, ciliary dysfunction, or various immunodeficiencies (Slavin et al 2005).

\section{Treatment}

There are no definitive guidelines for the treatment of CRS largely due to the lack of consensus on the etiology of the disease (Benninger et al 2003). CRS has commonly been treated with courses of oral antibiotics, but lack of clinical evidence for their efficacy in CRS makes this therapy controversial unless the patient is suffering from a superimposed acute bacterial inflammation upon the underlying chronic inflammatory condition (Slavin et al 2005). Unnecessary use of antibiotics in CRS may also

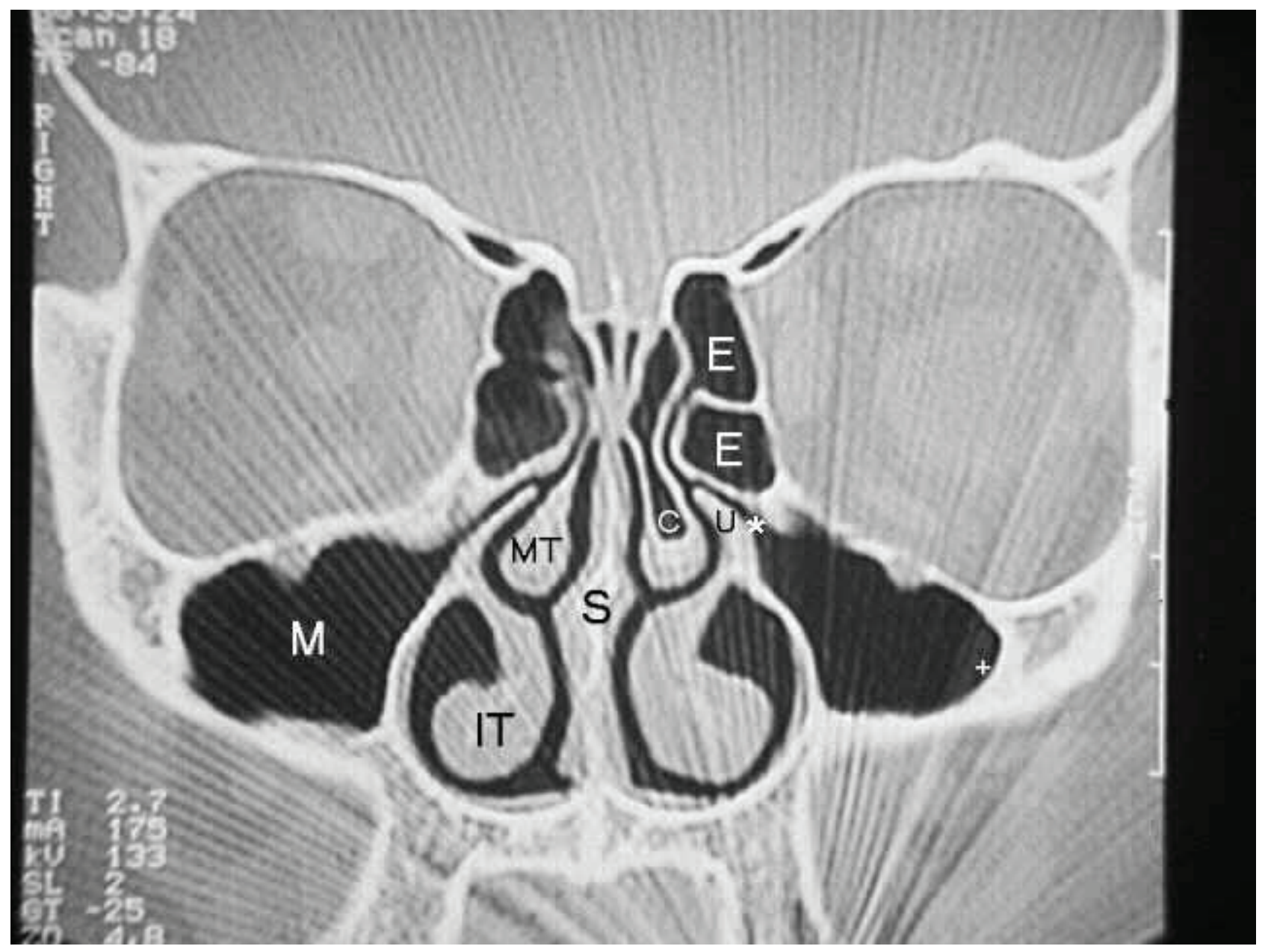

Figure I CT scan of normal sinuses (Tichenor 2006).

Note: +, border of maxillary sinus; *, maxillary sinus ostium; C, concha bullosa; CT, computed tomography; E, ethmoid sinuses; IT, inferior turbinate; MT, middle turbinate; $\mathrm{S}$, septum; $\mathrm{U}$, uncinate process. 


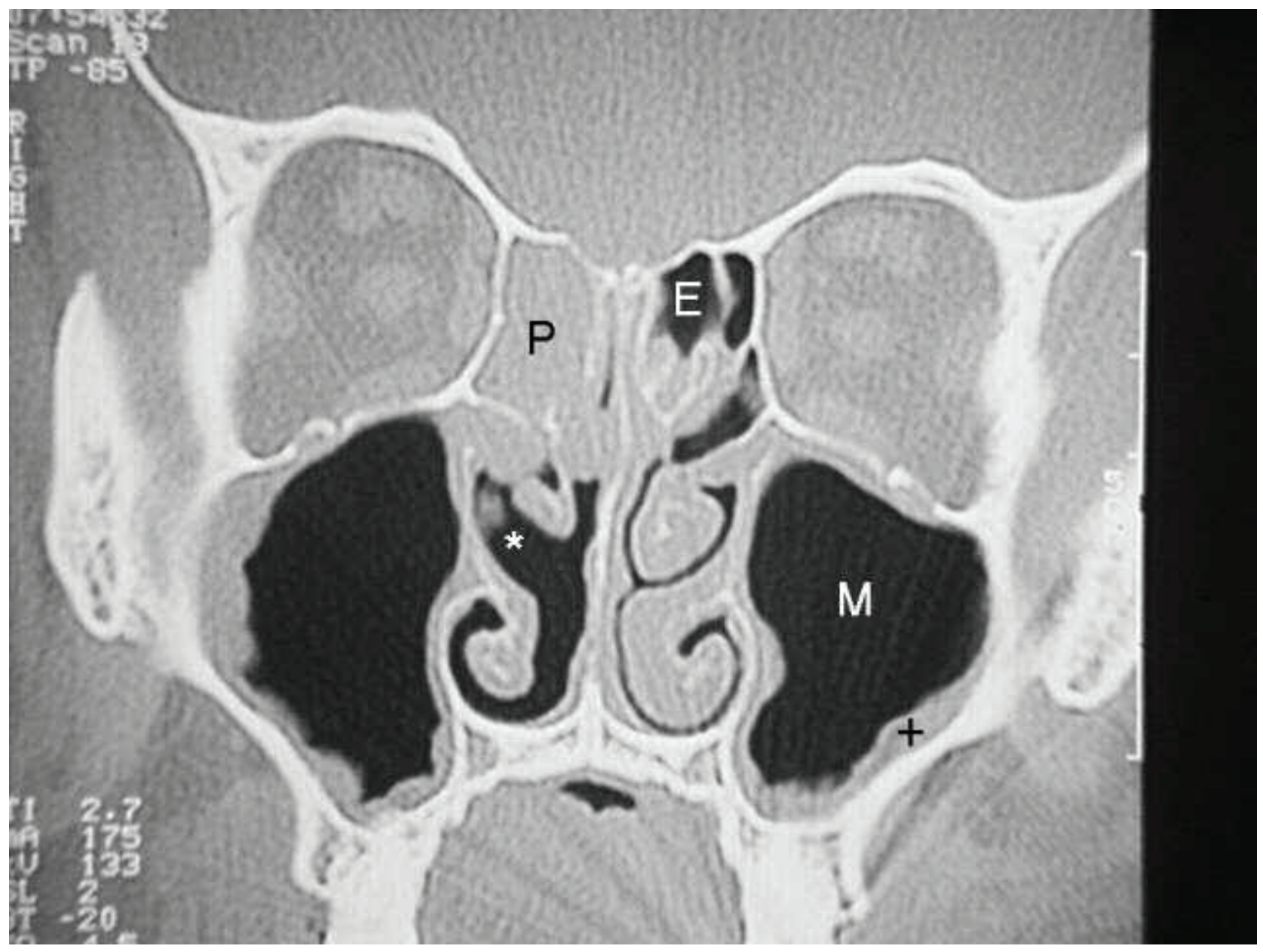

Figure 2 CT scan of sinuses with sinusitis (Tichenor 2006).

Note: +, thickening of the maxillary sinus; *, middle meatus; CT, computed tomography; E, ethmoid sinuses; M, maxillary sinus; O, maxillary sinus ostium; P, polyp.

contribute to the problem of antibiotic-resistant bacteria. There has been some use of inhaled topical antibiotics for CRS; a few uncontrolled studies have reported efficacy with these treatments, but controlled trials are needed to demonstrate effectiveness of this therapy (Slavin et al 2005).

Antihistamines are not recommended for CRS, unless the patient has an accompanying allergic rhinitis. Also, although topical and oral decongestants are often used, side effects such as rhinitis medicamentosa (with topical agents) and hypertension (with oral agents) can occur (Slavin et al 2005). Intranasal corticosteroids have been shown to relieve symptoms in CRS, but it is unclear if this is due to simply a decrease in nasal congestion or to decreased inflammation in the sinuses themselves. There is evidence that corticosteroids (topical and oral) are effective against nasal polyps (Slavin et al 2005). Studies have also shown that irrigation with hypertonic saline alleviates symptoms in CRS patients (Heatley et al 2001; Slavin et al 2005).

When there is documented underlying disease in CRS, treatment of the underlying condition may help relieve CRS symptoms as well. For example, intravenous immunoglobulin (IVIG) therapy should be given to patients with immunodeficiency, to prevent serious complications due to CRS (subperiosteal and intracranial abscesses, meningitis, sepsis, and death). Aspirin desensitization is recommended in patients with aspirin-exacerbated airway disease (Slavin et al 2005).

Surgery has long been a treatment of choice for persistent CRS, and with the advent of endoscopy, most surgeries are now minimally invasive. The main point of endoscopic sinus surgery (ESS) is to clear blockage and ensure patency of the osteomeatal complex (OMC) - the common drainage 
site of the frontal, maxillary, and anterior ethmoid sinuses (Witterick and Kolenda 2004), which often plays a role in perpetuation of the disease (Slavin et al 2005). Although studies have shown positive outcomes from surgery, with subjective improvement ranging from $70 \%$ to $98 \%$ of patients, surgery does not necessarily cure the disease and should be considered as an adjunct to medical therapy. Medical treatment is often still required after surgery, but possibly less frequently (Witterick and Kolenda 2004).

With current evidence pointing to the fungal etiology of CRS, there has been a trend towards treatment of CRS with topical antifungal medication, namely Amphotericin B $(\mathrm{AmB}) . \mathrm{AmB}$ is a natural polyene antifungal that is not absorbed through the gastrointestinal tract. AmB binds to ergosterol, a component of cell walls of most fungi, leading to formation of ion channels and cell death; AmB may also act secondarily through oxidative damage to fungal cell membranes through creation of free radicals from its own oxidation (Groll et al 2003). It is hypothesized that topical intranasal application of AmB can decrease the fungal load in the sinonasal region, thereby decreasing the local eosinophilic inflammatory reaction to fungal antigens seen in many CRS patients (Ponikau, Sherris, Weaver, et al 2005). The toxicity of intravenously administered AmB has been well characterized at much higher concentrations than are administered intranasally. There is clear evidence that AmB is poorly absorbed through the gut when ingested orally, therefore there is little or no potential for systemic exposure to the drug when administered by the topical intranasal route (Wise et al 1982; AmB PI 1996).

Although topical intranasal AmB has been considered by some as a controversial therapy, there is increasing evidence for the efficacy and safety of this antifungal drug for CRS patients. In a 2002 pilot study, $75 \%$ of CRS patients had improvement of symptoms, and 75\% showed improvement on endoscopic exam (Ponikau et al 2002). In a study of 74 patients with persistent nasal polyposis despite saline lavage and corticosteroid spray, addition of intranasal AmB was associated with complete disappearance of polyps in 39\% of patients (Ricchetti et al 2002).

A randomized controlled study of CRS patients with polyposis showed no improvement on CT and slightly worse symptom scores for AmB patients (Weschta et al 2004). However, this study excluded anyone with a clinical suspicion of AFRS based on the Brent-Kuhn criteria (Weschta et al 2004), the diagnostic criteria proposed for AFRS. In the original Brunt-Kuhn criteria for allergic fungal sinusitis (AFS), one needed positive allergy testing, the presence of fungi on histology or culture, eosinophilic "allergic mucin", and the presence of positive evidence on CT scan with nasal polyps. In the evidence based on our studies, CRS does not require positive allergy testing (ie, less than $50 \%$ of our patients had immunologic evidence of allergy). These criteria have been demonstrated to be present in the majority of CRS patients, placing the AFRS subentity into question (Ponikau et al 1999).

More recently, a randomized controlled trial was conducted with 30 CRS patients. This study demonstrated improvement in CRS on CT scan in the AmB group at 6 months, with a mean decrease in mucosal thickening of $8.8 \%$ compared with a mean increase of $2.3 \%$ for the placebo group ( $\mathrm{p}=0.03$ ). In addition, $70 \%$ of the AmB patients had improved endoscopy scores, and 9 out of $10 \mathrm{AmB}$ patients had improvement of symptoms as measured by SNOT-20 (compared with 6 of 14 placebo patients). The only adverse event due to AmB was the feeling of burning at the time of administration in 2 patients (Ponikau, Sherris, Weaver, et al 2005).

The majority of these studies suggest that $\mathrm{AmB}$ is an effective means of decreasing mucosal inflammation and improving symptoms in CRS patients. This positive effect occurs, in all likelihood, by decreasing the fungal antigen load, which in turn "shuts off" the eosinophilic response. It appears that the initiation of the immunologic response is the result of new hyphae, new spores, and this takes time for the reaction to be modulated and reduced. If there are antigenic portions of the fungi still present, it would initiate the continuation of the immunologic response. Destroying the fungi and reducing the amount of new hyphae and spores in turn reduces the immunologic response of the body in sensitized individuals. Therefore, this reduces the degranulation of eosinophils and reduces the eosinophilic population. Usually this reaction stops within a three month period.

\section{Conclusion}

AmB is safe and generally well tolerated by patients because is a topical therapy with minimal absorption. AmB should therefore be considered as a first-line therapy in CRS prior to considering surgical intervention. In patients with severe and extensive nasal polyps, it is best to operate and surgically remove the polyps and the mucus with its toxic MBP. Surgical removal of obstructing polypoid disease allows post-surgical access for the use of topical intranasal AmB to prevent and minimize disease recurrence. The endoscopic surgery to remove polyps with antrostomy allows AmB to 
gain access to the affected sinuses. Patients without prior history of surgery or severe stage 4 polyposis might not have as much benefit.

As the definitions and terms used to describe types of CRS have changed, so have the theories behind the causes of this common yet complex disease. With the currently small amount of knowledge on the etiology and pathogenesis of CRS, it remains a priority to treat the symptoms of CRS by decreasing inflammation and obstruction, either medically or surgically. However, as evidence emerges to support the role of fungi in the pathogenesis of CRS, intranasal topical antifungal treatment can be considered as an early line of therapy to safely relieve the symptoms of CRS and, in the post-surgical patient, to prevent or delay recurrence.

\section{References}

[AmB PI] 1996. Fungizone oral suspension (Amphotericin B) (complete prescribing information) Bristol-Myers Squibb Co.

Benninger MS, Ferguson BJ, Hadley JA, et al. 2003. Adult chronic rhinosinusitis: definitions, diagnosis, epidemiology, and pathophysiology. Otolaryngol Head Neck Surg, 129(3 Suppl):S1-32.

Frigas E, Loegering DA, Gleich GJ. 1980. Cytotoxic effects of the guineapig eosinophil major basic protein on tracheal epithelium. Lab Invest, 42:35-43.

Gleich GJ, Adolphson CR, Leiferman KM. 1993. The biology of the eosinophilic leukocyte. Annu Rev Med, 44:85-101.

Gosepath J, Brieger J, Vlachtsis K, et al. 2004. Fungal DNA is present in tissue specimens of patients with chronic rhinosinusitis. Am J Rhinol, 18(1):9-13.

Groll AH, Gea-Banacloche JC, Glasmacher A, et al. 2003. Clinical pharmacology of antifungal compounds. Infect Dis Clin North Am, 17:159-91, ix.

Hamilos DL, Lund VJ. 2004. Etiology of chronic rhinosinusitis: the role of fungus. Ann Otol Rhinol Laryngol Suppl, 193:27-31.

Heatley DG, McConnell KE, Kille TL, et al. 2001. Nasal irrigation for the alleviation of sinonasal symptoms. Otolaryngol Head Neck Surg, 125:44-8.
Lethbridge-Çejku M, Rose D, Vickerie J. 2006. Summary health statistics for U.S. Adults: National Health Interview Survey, 2004. National Center for Health Statistics. Vital Health Stat, 10(228).

Lethbridge-Çejku M, Schiller JS, Bernadel L. 2004. Summary health statistics for U.S. Adults: National Health Interview Survey, 2002. National Center for Health Statistics. Vital Health Stat, 10(222).

Meltzer EO, Hamilos DL, Hadley JA, et al. 2004. Rhinosinusitis: establishing definitions for clinical research and patient care. J Allergy Clin Immunol, 114(6 Suppl):155-212.

Motojima S, Frigas E, Loegering DA, et al. 1989. Toxicity of eosinophil cationic proteins for guinea-pic tracheal epithelium in vitro. Am Rev Res Dis, 139:801-5.

Ponikau JU, Sherris DA, Kephart GM, et al. 2005. Striking deposition of toxic eosinophil major basic protein in mucus: implications for chronic rhinosinusitis. J Allergy Clin Immunol, 116:362-9.

Ponikau JU, Sherris DA, Kephart GM, et al. 2003. Features of airway remodeling and eosinophilic inflammation in chronic rhinosinusitis: is the histopathology similar to asthma? J Allergy Clin Immunol, 112:877-82.

Ponikau JU, Sherris DA, Kern EB et al. 1999. The diagnosis and incidence of allergic fungal sinusitis. Mayo Clin Proc, 74:877-84.

Ponikau JU, Sherris DA, Kita H, et al. 2002. Intranasal antifungal treatment in 51 patients with chronic rhinosinusitis. J Allergy Clin Immunol, 110:862-6.

Ponikau JU, Sherris DA, Weaver A, et al. 2005. Treatment of chronic rhinosinusitis with intranasal amphotericin B: a randomized, placebocontrolled, double-blind pilot trial. J Allergy Clin Immunol, 115:125-31.

Ricchetti A, Landis BN, Maffioli A, et al. 2002. Effect of anti-fungal nasal lavage with amphotericin B on nasal polyposis. J Laryngol Otol, 116:261-3.

Sasama J, Sherris DA, Shin SH, et al. 2005. New paradigm for the roles of fungi and eosinophils in chronic rhinosinusitis. Curr Opin Otolaryngol Head Neck Surg, 13:2-8.

Slavin RG, Spector SL, Bernstein IL, et al. 2005. The diagnosis and management of sinusitis: a practice parameter update. J Allergy Clin Immunol, $116(6$ Suppl):S13-47.

Tichenor WS. 2006. Sinusitis for physicians 1998-2006 [online]. Accessed on 15 August 2006. URL: http://www.sinuses.com/ search_site.cgi? fname $=$ md.htm $\& d b=s \& s k w=$ diagnosis $\&$ method $=$ and

Weschta M, Rimek D, Formanek M, et al. 2004. Topical antifungal treatment of chronic rhinosinusitis with nasal polyps: a randomized, double-blind clinical trial. J Allergy Clin Immunol, 113:1122-8.

Wise GJ, Kozinn PJ, Goldberg P. 1982. Amphotericin B as a urologic irrigant in the management of noninvasive candiduria. $J$ Urol, 128:82-4.

Witterick IJ, Kolenda J. 2004. Surgical management of chronic rhinosinusitis. Immunol Allergy Clin North Am, 24:119-34. 
